\title{
LYMPHOME DE BURKITT PÉDIATRIQUE À RÉVÉLATION ORL : QUELLE PRISE EN CHARGE EN MILIEU SUB SAHÉLIEN ? RÉFLEXION SUR TROIS CAS
}

\author{
B. TOGO, M.A. KEITA *, M. DIALLO ${ }^{* *}$, F. TRAORÉ , C.B. TRAORÉ ${ }^{* *}$, A. AG MOHAMED*, M. KEITA \\ SERVICE DE PÉDIATRIE, UNITÉ D'ONCOLOGIE. HÔPITAL GABRIEL TOURÉ BAMAKO.MALI \\ *SERVICE ORL, HÔPITAL GABRIEL TOURÉ BAMAKO. MALI \\ **SERVICE DE RADIOLOGIE, HÔPITAL GABRIEL TOURÉ BAMAKO. MALI \\ ${ }^{* * *}$ SERVICE ANATOMOPATHOLOGIE INRSP BAMAKO. MALI
}

\begin{abstract}
Le lymphome de Burkitt dans sa forme Africaine peut se manifester sous des signes d'emprunt ORL à type de tuméfaction orbito-maxillo-faciale. Le diagnostic peut être fait par les ORL à travers un faisceau de signes cliniques et une cytoponction. La prise en charge se fait dans des unités d'oncologie médicale ou le bilan d'extension et le lancement de la chimiothérapie sont des impératifs incontournables.

Actuellement cette tumeur à potentiel très agressif répond à une poly chimiothérapie bien conduite.

Mots-clés : Lymphome de Burkitt, cancer, voies aérodigestives supérieures, oncologie pédiatrique
\end{abstract}

Burkitt's lymphoma in its African form can be expressed with a maxillofacial swelling. The diagnosis can be made through a set of clinical signs and a fine needle aspiration cytology. The management of this disease is well done in an oncology department, where extension status and chemotherapy are essential requirements. This aggressive lymphoma responds well to polychemotherapy.

Keywords: Burkitt's lymphoma, upper aerodigestive tract cancer, pediatric oncology.

\section{INTRODUCTION}

Faisant partie du groupe des LMNH, le Lymphome de Burkitt (LB) est un lymphome malin à cellule B mature d'aspect très caractéristique décrit en 1958 par Denis Burkitt à Kampala (Ouganda). Trois formes existent : I'une est endémique (Afrique noire), liée au virus Epstein Barr (EBV) ; l'autre est sporadique (occidentale), non liée à l'EBV et la forme liée à l'HIV. Cette tumeur très proliférante peut envahir la moelle osseuse, les méninges et les séreuses. C'est le plus fréquent des lymphomes malins de l'enfant (35 à $45 \%$ des lymphomes). II est beaucoup plus rare chez l'adulte (1).

Le lymphome de Burkitt au niveau de la sphère ORL garde les caractères communs à l'ensemble des lymphomes et des caractères particuliers. Les caractères communs sont la possibilité d'extension à distance, et la réponse habituelle à la chimiothérapie. Les caractères particuliers liés à la localisation ORL impliquent certaines conduites dans la recherche de l'extension initiale, dans le traitement et dans la surveillance (2).

Actuellement un traitement adapté couplé à une surveillance clinique et biologique vigilante donne au lymphome de Burkitt une survie globale de $90 \%$ même à un stade avancé (3).

Notre but à travers ces observations est de rappeler la clinique de cette tumeur fréquente de l'enfant en Afrique subsaharienne et de montrer les résultats de nos protocoles chimiothérapiques basés sur ceux du LMB89 et sur celui de Hesseling au Malawi avec des drogues fournies par le Groupe Franco-Africain d'Oncologie Pédiatrique.

\section{OBSERVATIONS}

\section{Observation $\mathrm{N}^{\circ} \mathbf{1}$ :}

S.C, 8 ans, élève, quatrième enfant d'une fratrie de 7 , a été admis en ORL le 6 novembre 2006 pour exophtalmie gauche et tuméfaction cervico-faciale homolatérale.

A l'admission il s'agissait d'un garçonnet de 8 ans présentant depuis deux mois et demi une exophtalmie non fébrile et non axile avec un important chémosis entraînant une occlusion palpébrale. A cette exophtalmie était associée une infiltration des tissus mous de l'hémiface homolatérale. On notait en plus une poly adénopathie en sous maxillaire et jugulodigastrique, très dure à la palpation, non mobilisable et sans note douloureuse.

Le reste de l'examen retrouvait un garçon amaigri pesant dix sept kilogrammes avec une surface corporelle à 0,7 m2. L'examen abdominale objectivait une masse de la région épigastrique de taille $15-10 \mathrm{~cm}$, non douloureuse à la palpation, ferme, de contour régulier et à surface lisse. Devant ce tableau, nous avons réalisé : 
- un bilan du malade avec une recherche de pathologies infectieuses sous jacentes (tuberculose, hépatite $B$ et VIH) négative ;

- un bilan de la maladie comportant : une cytoponction ganglionnaire, une ponction médullaire, la TDM cérébrale, la cytologie du LCR, un hémogramme, une goutte épaisse, une Radiographie du thorax de face et une échographie abdominale.

- Un ionogramme sanguin, la LDH, uricémie, furent réaliser aussi.

La radiographie du thorax mettait en évidence un élargissement du médiastin supérieur gauche. L'échographie abdominale montrait une hépato-splénomégalie homogène et des paquets d'adénopathies profondes. L'ionogramme sanguin était normal.

L'hémogramme mettait en évidence une discrète anémie normochrome.

La lecture cytologique a retrouvé des cellules lymphoïdes de taille moyenne à grande, au rapport nucléo-cytoplasmique et index mitotique élevés, et un cytoplasme basophile renfermant des vacuoles. On notait la présence de macrophages au sein de cette figure rappelant un ciel étoilé du lymphome de Burkitt. Le patient a été transféré en oncologie pédiatrique

Le patient a reçu trois cures de chimiothérapie à une semaine d'intervalle suivie de quatre cures de rattrapage à 18 jours d'intervalles.

Le patient a reçu une dose de $680 \mathrm{mg}$ d'Endoxan en IVD associé à une injection intrathecale de ledertrexate (15 $\mathrm{mg}$ ) et de methylprednisolone $(15 \mathrm{mg})$. Le patient était en rémission partielle au bilan du J21, ce qui a motivé les cures de rattrapage :

- 2 cures d'induction COPM (CyclophosphamideOncovin- Prednisone- Methotrexate à haute dose)

- 2 cures de consolidation CYM (Cytarabine Cyclophosphamide- Methotrexate à haute dose). La rémission complète fut obtenue à la fin de la seconde cure COPM et se poursuit.

\section{Observation $\mathrm{N}^{\circ} \mathbf{2}$ :}

A.D, 6 ans, M, a été reçu le 19/09/06 dans le service ORL pour tuméfaction maxillo-cervicale gauche. L'histoire de la maladie a retrouvé une notion d'adénopathies inguinales apparues sept mois plutôt et qui aurait disparu sous antibiothérapie non spécifié. Mais, un mois après le patient a présenté une asthénie permanente. En plus de cette asthénie, l'augmentation progressive de la taille de la tumeur a motivé la demande de soins actuels.

A l'admission le patient présentait un chapelet d'adénopathies maxillo-cervicale gauche, dure à la palpation avec une peau en regard normale, non douloureuse. Le reste de l'examen ORL a été normal.

A l'examen général, le patient était amaigri avec des côtes saillantes. II pesait $14 \mathrm{~kg}$

(-2DS) avec une surface corporelle à 0,61 m2.La pâleur conjonctivale était franche. L'examen cardio-pulmonaire était normal, il n'y avait pas d'organomégalie.

Nous avons procédé à un bilan du malade : un hémogramme (anémie normochrome à $8 \mathrm{~g} / \mathrm{dl}$ ), une goutte épaisse (positive) et une recherche de $\mathrm{VIH}$ et de tuberculose négatives.

Le bilan de la maladie a été fait : la radiographie du thorax et l'échographie abdominale n'ont révélé aucune localisation secondaire.

La cytologie a retenu un lymphome de Burkitt et le transfert sur l'unité d'oncologie pédiatrique a été fait.

Le patient a reçu trois cures de chimiothérapie à une semaine d'intervalle.

-une dose de $560 \mathrm{mg}$ d'Endoxan en IVD associé à une injection intrathecale de ledertrexate $(15 \mathrm{mg})$ et de methylprednisolone $(15 \mathrm{mg})$. Le patient était en rémission partielle au bilan du J21.

Il a présenté une aplasie médullaire profonde à la fin de la fin de la troisième cure. II en est décédé à la suite des complications infectieuses, dans un tableau de syndrome pseudo-occlusif avec une fièvre en plateau à $38^{\circ} 5 / 10$ ème.

\section{Observation $\mathrm{N}^{\circ} 3$ :}

DS, 6 ans, sexe F, a été admise en hospitalisation ORL le $5 / 11 / 07$ pour masse sous orbitaire droite.

L'interrogatoire a retrouvée une notion de masse sous orbitaire droite installée de façon progressive depuis quatre mois sans contexte douloureux associée à une altération progressive de l'état général. Les antécédents tant personnels que familiaux de la patiente ont été sans particularité.

L'examen ORL a trouvé une masse rénitente de la région sous orbitaire droite de la taille d'un œuf de poule, non douloureuse, avec aspect d'infiltration des régions adjacentes.

Le reste de l'examen ORL a été normal ainsi que l'examen cardio-pulmonaire.

L'examen général a trouvé une patiente cachectique, pesant 12kgs (-3 DS) pour une surface corporelle de $0,54 \mathrm{~m} 2$. On notait une pâleur conjonctivale et une volumineuse ascite. On notait une douleur du membre inferieur gauche réveillée par la mobilisation.

Nous avons procédé à un bilan de la maladie et du malade qui a retrouvé une discrète anémie microcytaire normochrome à $10 \mathrm{~g} / \mathrm{dl}$ et une goutte épaisse positive. La TDM maxillo-faciale a mise en évidence une masse de densité homogène occupant la région sous orbitaire sans lyse osseuse.

La TDM cérébrale a retrouvé un processus de la fosse temporale droite en faveur d'un méningiome ou d'une métastase. La radiographie du thorax de face a été normale. L'échographie abdominale a retrouvé une image orientant vers une importante coulée ganglionnaire abdominale avec infiltration pancréatique et splénique et un 
épanchement liquidien intra-péritonéal pouvant faire évoquer soit un processus lymphomateux soit des lésions secondaires.

La cytologie a objectivé des plages de cellules arrondies ou ovalaires de taille moyenne avec un noyau dense hyperchromatique, un cytoplasme peu abondant. Ces cellules étaient parsemées de rares cellules macrophagiques. L'Aspect cytologique a été très suspect d'un lymphome de Burkitt.

Nous avons retenu le diagnostic de Lymphome de Burkitt Facial et la patiente a été admise en oncologie pédiatrique.

La patiente a reçu trois cures de chimiothérapie à une semaine d'intervalle suivie de quatre cures de rattrapage à 21 jours d'intervalles.

La patiente a reçu une dose de $480 \mathrm{mg}$ d'Endoxan en IVD associé à une injection intrathecale de ledertrexate (15 $\mathrm{mg}$ ) et de methylprednisolone (15 mg). Un effet objectif fut obtenu au bilan du J21, ce qui a motivé les cures de rattrapage :

- 2 cures d'induction COPM (CyclophosphamideOncovin- Prednisone- Methotrexate à haute dose)

- 2 cures de consolidation CYM (Cytarabine Cyclophosphamide- Methotrexate à haute dose). La rémission complète fut obtenue à la fin de la première cure COPM en fin janvier 07 et se poursuit.

\section{DISCUSSION}

La distribution anatomique varie selon l'endémicité du lymphome. Deux de nos patients comme décrit dans le Burkitt africain (1), ont présenté des localisations préférentielles au niveau du massif facial sous forme de volumineuses tumeurs avec déformation maxillo-orbito-malaire. Ils avaient un âge moyen de 6 ans et étaient de sexe masculin. Dans la littérature occidentale l'enfant, de sexe masculin, vers l'âge de 3 ans est le plus souvent atteint. Les autres localisations intéressent les organes génitaux primaires, les reins, les poumons et le SNC.

Cependant nous sommes en accord avec les constats africains ou il semble que l'âge moyen est de 6-7 ans $(4,5,6,7)$.

La prédominance chez le garçon est un constat établi (8). II semble également dans cette forme que les localisations abdominales deviennent plus fréquentes avec l'âge. Dans une étude en Ouganda, $70 \%$ des enfants de moins de 5 ans avaient une localisation mandibulaire, alors que celle-ci n'était présente que dans $25 \%$ des cas après 14 ans (9).

Dans le Burkitt non africain, la distribution anatomique est différente. La majorité des patients présente une tumeur abdominale. Les ganglions sont souvent atteints notamment abdominaux et cervicaux. Les localisations extraganglionnaires ORL se retrouvent dans $24 \%$ des cas (Pattee, 1980) et intéressent surtout l'amygdale et le cavum (1).
Cependant tous les lymphomes de Burkitt africain ou non, sont semblables sur le plan anatomo-pathologique, immunologique et chromosomique (5).

Nous sommes en accord avec la littérature qui estime que la variété des signes cliniques et des localisations tumorales simultanées sont des caractéristiques bien spécifiques de la maladie de Burkitt probablement en rapport avec la déficience immunitaire, contrairement aux autres cancers ou l'extension a plutôt tendance à s'échelonner dans le temps. Le début est caractérisé par la forme tumorale isolée ou associée à d'autres signes ou à d'autres événements tels que des extractions dentaires intempestives (5). Cependant comme rapporté par notre observation $\mathrm{N}^{\circ} 2$ il est dit (4) que l'atteinte ganglionnaire cervicale peut, à elle seule, résumer le tableau clinique en dehors de toute manifestation maxillo-faciale ou abdominale.

Notre diagnostic a été fondé sur l'étude des frottis des diverses cytoponctions réalisées au sein des masses tumorales. Dans un cas nous avons eu recours à l'histologie par la lecture d'une biopsie ganglionnaire.

Ce diagnostic est basé ailleurs sur la cytologie mais aussi sur l'histologie $(4,9)$. La tumeur de Burkitt est un lymphome à cellules $B$ mature, avec un aspect histologique caractéristique en ciel étoilé causé par la présence de phagocytes mûrs, grands et clairs, dans un environnement de cellules lymphoïdes monomorphes au rapport nucléocytoplasmique élevé et contenant des vacuoles. Le cytoplasme est basophile avec un indice mitotique élevé. C'est une maladie d'évolution rapide, avec un temps de duplication estimé de 24 à 66 heures (10).

Ce diagnostic dans les centres plus équipés établi le phénotype et recherche un réarrangement de c-myc en cytogénétique ou en biologie moléculaire. Ce c-myc a été l'un des premiers oncogène reconnu en pathologie tumorale. Les auteurs sont unanimes sur la grande agressivité du LB et trouve comme essentiel de faire rapidement un bilan d'extension à la recherche de localisations notamment neuromeningée et médullaire d'où l'intérêt du myélogramme.

Pour nos patients nous avons réalisé un bilan de la maladie (échographie abdomino-pelvienne, radiographie thoracique, TDM maxillo-faciale et cérébrale) à la recherche d'extension. Ce qui a permis d'isoler une extension viscérale chez deux de nos patients et une suspicion de localisation cérébrale associé dans un cas, lesquelles extensions associées à la tumeur maxillo-faciale donne le stade III et IV selon la stadification clinique de Murphy (tableau I). La prédominance du stade III et IV de Murphy s'expliqueraient selon la littérature par le délai tardif de consultation. 


\section{Stades cliniques d'extension de la maladie de Burkitt selon la classification de Murphy (1980)}

\section{Stade1}

- tumeur unique extraganglionnaire

- ou un seul territoire ganglionnaire à l'exclusion du médiastion et de l'abdomen

Stade 2

- Tumeur unique extraganglionnaire avec extension ganglionnaire régionale

- Deux tumeurs extraganglionnaires avec ou sans atteinte ganglionnaire régionale du même côté du diaphragme - Tumeur primitive du tube digestif avec ou sans atteinte des ganglions mésentériques satellites

Stade 3

- deux tumeurs extraganglionnaires de part et d'autre du Diaphragme

- deux ou plusieurs atteintes ganglionnaires de part et d'autre du diaphragme

- toute tumeur primitive abdominale dépassant le tube digestif et les ganglions mésenteriques

- toute atteinte vertébrale avec ou sans autre localisation Stade 4

- toute atteinte déjà mentionnée avec envahissement du système nerveux central ou atteinte de la moelle osseuse - atteintes neuromenigées ou médullaires initiales quelles que soient les autres localisations.

Tableau I: Stadification clinique de Murphy

Au début du traitement chimiothérapique, il est rapporté que l'évolution peut être marquée par la survenue de troubles métaboliques parfois mortels constituant le syndrome de lyse tumorale aigue (Cohen 1980) se manifestant par une hyperkaliémie, une hyperphosphoremie, une hyper uricémie et une hypocalcémie. Le risque de survenue de ce syndrome est proportionnel au volume tumoral initial et au taux sérique de la lacticodéhydrogénase (Arseneau, 1975).

Nous avons complété le bilan de nos malades par la créatinémie et l'uricémie et un ionogramme répété en cours de traitement pour déceler les risques sus-cités (1).

Par contre nous n'avons pas pu réaliser la sérologie EBV, or on estime la fréquence de cette association autour de 80 à 100\%.(7). L'étape finale commune de la pathogénie du LB fait intervenir l'activation d'un oncogène cellulaire cité plus haut le c-myc.

Quand à la stratégie thérapeutique comme rapportée par la littérature $(2,4,6,11,12)$ nous avons opté pour l'utilisation polychimiothérapie intensive et de durée brève. Nous avons pour chaque patient réalisé 3 cures initiales à une semaine d'intervalle. La suite de la chimiothérapie était conditionnée au bilan du J21.Une réponse insuffisante après trois cures, nécessitait une chimiothérapie de rattrapage. Le cyclophosphamide et le methothrexate constituaient la base de notre traitement, comme dans d'autres associations utilisées actuellement dans le traitement des lymphomes de Burkitt.

Quand aux traitements adjuvant rapporté dans la littérature (6) nous avons eu d'énormes difficultés à gérer les aplasies médullaires consécutives à la chimiothérapie, vu la pauvreté des familles. Les mesures d'hygiène à type d'isolement en chambre individuelle n'ont pu être exécutées et la disponibilité d'antifongique et de décontamination digestive à l'aide d'antibiotique n'a pas été systématique.

L'évolution dans un cas a été dramatique aboutissant rapidement au décès. Dans les deux autres cas nous avons été confrontés à des effets secondaires et à des difficultés liées à l'impossibilité de suivi biologique répété de nos patients sous chimiothérapies suite à des problèmes structurelles.

Ailleurs il est rapporté que l'évolution est caractérisée par la rapidité de la croissance tumorale parfois responsable de complications mécaniques pouvant être létales (obstruction des VADS, Insuffisance Rénale aigue). Les complications précoces et gravissimes soulignent l'intérêt d'un diagnostic et d'un traitement urgent.

A travers la littérature il est dit que la réponse immédiate au traitement représente un facteur pronostique essentiel : tous les patients qui ne répondent pas favorablement à la première chimiothérapie décèdent dans les mois qui suivent $(10 \%$ selon Ziegler, 1972) $(1,13)$. La majorité des patients entrent en rémission complète, mais un certain nombre présenteront des rechutes, soit précoces (3 premiers mois) caractérisées par la récidive tumorale au niveau du site primitif et par l'envahissement du SNC de mauvais pronostic ; soit tardives marquées par l'apparition de nouvelles localisations néoplasiques sans atteinte du SNC, de meilleur pronostic et chimiosensibles.

\section{CONCLUSION}

Le lymphome de Burkitt mérite d'être connu d'autant que les traitements actuels ont permis d'allonger le taux de survie à plus de $90 \%$. Cette pathologie dépistée tôt, permettra sous nos contrées de définir le schéma thérapeutique précocement. Cette démarche permettra de mettre en marche le clan familial pour faire face au coût de la chimiothérapie qui reste encore hors de portée de nos citoyens moyens. 


\section{REFERENCES}

1.Jan A, Vora K, Sandor JKB Sporadic Burkitt's lymphoma of the jaws: the essentials of prompt life-saving referral and Management $\mathrm{J}$ Can Dent Assoc 2005;71(3):165-8

2.Buffe P., Soubeyrand L. et Cudennec Y.F Les maladies tropicales en ORL EMC. (Paris, France), ORL, 20925 A10, 10-1986,10p.

3. Philip T, Bergeron C Le lymphome de Burkitt : un modèle pour la cancérologie pédiatrique Arch Pédiatr $2000 ; 7: 924-6$

4.Diop EM, Diouf R, Ndiaye IC, Tending G, Tall A, Toure S Maladies tropicales ORL Encycl Med Chir Oto-rhino-laryngologie 20925 A10, 2000,16p.

5. Rafaramino F, Maminirina RAM, Razafindrabe JAB, Rabarijaona L, Randriamampandry, Rakotobe $\mathrm{P}$ Le lymphome de l'enfant à Madagascar. Formes anatomo-cliniques, aspects thérapeutiques et évolutifs. Bull Soc Pathol Exot, 2001, 94,5,389-393.

6. Koffi KG, Bosson NM, Aka-Adjo M.A, Diop S, N'Dahtz, Ahmedou O, Sanogo I, Sangaré A. Résultats du traitement du lymphoma de Burkitt Africain Expérience du service d'hématologie clinique du CHU de Yopougon (abidjan) Médecine d'Afrique noire ;1997,44(12) :635-639.

7. Doumbé P, Mbakop A, Essomba mboumi MT, Obama MT, Kago I, Gonsu J,
Santiago M, Tetanye E Lymphome de Burkitt chez l'enfant au Cameroun : Aspects descriptifs et anatomocliniques Bull cancer 1997, 84(4) : 379-83.

8. Junquera Gutierrez LM, Albertos Castro J.M, Alonso Vaquero D, Vicente Rodriguez J.C, Lopes Arranz J.S Lymphome de Burkitt Americain de localisation mandibulaire Rev. Stomatol. Chir. Maxillofac 1994,95,5:355-358 9. Ribrag V, Bosq J Lymphomes de Burkitt,

Encycl Med Chir (Editions scientifiques et Médicales Elsevier SAS, Paris, tous droits reserves) Hématologie, 13-016 A50, 2002, 9p

10. Standefer JA, Mattow DE, Aufdemorte TB, Geiser CF, Gates GA Midfacial Burkitt's lymphoma Otolaryngol Head Neck Surg 1985, 93:262-7.

11. Madani A, Benhmiddoune L, Zafad S, Harif M, Quessar A, Benchekroun S Traitement du lymphoma de Burkitt de l'enfant par le protocole LMB89 à Casablanca Bull Cancer 2005 ; 92(2) :193-8.

12.Brugère J., Dumont J. et Charpy- Validire $P$ Lymphomes des VADS- Editions Techniques- EMC (Paris, France), ORL, 20957 A10, 1991, 4p.

13. Ziegler JL Burkitt lymphoma New Engl J Med 1981; 13: 735-41 